\title{
Recurrence risks in complex inheritance with special regard to pyloric stenosis ${ }^{1}$
}

\author{
J. M. LALOUEL, N. E. MORTON, C. J. MAcLEAN, AND J. JACKSON
}

From the Population Genetics Laboratory, University of Hawaii, Honolulu, Hawaii 96822; the Department of Preventive Medicine, School of Medicine, University of Mississippi Medical Center, Jackson, Mississippi 39216, U.S.A.

SUMMARY A large body of data on segregating families is used to generate specific recurrence risks conditional on sex and birth order for the best-fitting model of polygenes plus maternal effect. The. method is general for diseases of complex inheritance, and lies within the competence of any serious $\vec{A}$ genetic clinic. The question of whether consultees demand as much specificity should be subordinate to the question of whether counsellors are justified in providing less.

Genetic counselling is a transaction which includes specification of a recurrence risk for a particular disease in a particular family, using parameters from segregation analysis of a larger body of data. This problem has been solved for simple cases (Murphy and Mutalik, 1969), and the utility of computer programmes to determine specific risks has been demonstrated (Heuch and $\mathrm{Li}, 1972$ ). It remains to generalise these prototypes to more complex pedigrees and modes of inheritance.

As a first step we here apply the mixed model of Morton and MacLean (1974) to a large body of data on pyloric stenosis, a disease of obscure and presumably complex aetiology whose incidence varies widely with sex, birth order, and ethnic group. The mixed model subsumes polygenes, major loci, and common environment. Application of the mixed model illustrates the power and specificity which complex segregation analysis brings to prediction of recurrence risks.

\section{Pyloric stenosis}

Diagnosis of a hypertrophic muscular tumour of the pylorus presents several problems. The tumour may be present without causing symptoms at any time (Carter and Powell, 1954). Distinction from pylorospasm is not clear. For some authors 'pylorospasm exists more in the mind of the paediatrician than in the abdomen of the child' (Thomson and Gaisford, 1935), while others consider it a distinct entity

${ }^{1}$ This work was supported by Grant GM 17173 from the National Institutes of Health.

Received for publication 25 April 1977
(Craig, 1955). For Holt (1917) the great majority of cases of pylorospasm represent mild forms of organic pyloric stenosis. It is usually assumed that hypertrophy is a precursor of pylorospasm, though the $\Phi$ work of Heinsisch (1967) suggests that pylorospasm may induce hypertrophy.

Incidence of pyloric stenosis in Northern Euro ranges between 2 to 3 per thousand; it is lower Eastern Europe and even less among children of African or Asian descent (Leck, 1976). That incidence varies greatly with ethnic groups in a relatively homogeneous socioeconomic setting (Shim et al., $\stackrel{\AA}{\varnothing}$ 1970) has been taken by Dodge (1972b) as an indi- $\overrightarrow{\vec{A}}$ cation of the importance of a genetic susceptibility. 을 A decrease in incidence over time has been reported in Sweden (Wallgren, 1960) and Ireland (Dodge, 1975), but it may be that this trend reflects progress in early domiciliary medical treatment (Leck, 1976).

An essential feature of pyloric stenosis is that the male :female ratio among affected is found between 3 $4: 1$ and $5: 1$ in all populations, regardless of overall $\delta$ incidence (Leck, 1976); it cannot be explained by any simple mode of sex-linked inheritance, and Carter 0 (1961) suggested a sex-modified multifactorial background that can also account for the effect of sex of $\frac{\text { o }}{2}$ index patient on empirical risks to relatives (see for example Carter, 1972, 1976).

Evidence of higher incidence among first-born has $N$ been reported by various authors (e.g. McKeown et al., 1951a), with no or little maternal age effect, $\stackrel{\omega}{\sigma}$ though some reports did not show such birth order effect (see Leck, 1976). Bias towards higher social

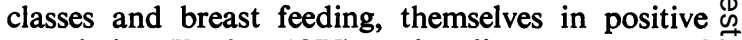
association (Dodge, 1975), and earlier occurrence of 
symptoms among infants fed on a 3-hourly schedule than among those fed on a 4-hourly schedule (Gerrard et al., 1955) have also been reported. A significant increase of emotional stress during the last trimester of pregnancy in women giving birth to an affected child suggests a maternal effect (Dodge, 1972a); it could explain association with primogeniture (Morris, 1968), though association with feeding patterns may incriminate some degree of learning. A maternal effect has also been suggested by Carter (1972) to explain the large increase in incidence of affection among children of affected mothers. Experimental production of hypertrophic pyloric stenosis in puppies by injection to the mother of pentagastrin (Dodge, 1970), confirmed by Karim et al. (1974), led Dodge (1972a) to suggest that a psychosomatic mechanism might lead to the transplacental transmission of a humoral factor, possibly gastrin, as it has been shown to cross the placenta experimentally (Bruckner et al., 1970). Another observation pointing to a possible maternal effect was reported by Dodge (1974): 'though the infants deviated significantly from the general population in respect of their $\mathrm{ABO}$ blood groups, with a deficiency of group A, their mothers showed an even greater divergence from the control distribution', and the effect of ABO blood group on fat absorption (Beckman, 1969) was taken by Dodge as suggestive evidence of the role of perturbation of the mechanism of gastric emptying in the development of pyloric stenosis.

Of the various disorders that may present some association with pyloric stenosis (Dodge, 1972b), only duodenal ulcer, also associated with blood group $\mathrm{O}$, seems significant (Cockayne and Penrose, 1943; Dodge 1970).

All this evidence points to a complex mechanism responsible for pyloric stenosis, implicating both genetic and environmental factors. No Mendelian model of inheritance can account for the familial aggregation observed, and interestingly Carter (1961) suggested a model of inheritance quite analogous to the mixed model.

\section{Segregation analysis}

FAMILIAL DATA SUBMITTED TO SEGREGATION ANALYSIS

Data for the present study, drawn from published material, consist of three samples, all from Great Britain over approximately the same period.

Carter and Evans (1969) reported a family study of pyloric stenosis based on two series of index patients treated surgically at The Hospital for Sick Children, London. The main series consisted of boys treated from 1920 to 1939 and girls treated from 1920 to 1949. They were traced in adult life, and therefore gave information on offspring and nephews and nieces, as well as sibs and first cousins. The supplementary series of boys treated from 1953 to 1962 and girls treated from 1950 to 1965 gave information on sibs, aunts and uncles, and first cousins. For the purpose of the present study, these samples consisted of 967 sibships drawn through an affected sib and 436 sibships drawn through an affected parent.

Cockayne and Penrose (1943) reported sibships of 212 visited families from London. The propositi had mostly been treated in two London hospitals between 1920 and 1935.

McKeown et al. (1951b) presented data which included all diagnosed cases domiciled in Birmingham and admitted to Birmingham hospitals during the 10-year period 1940-49: 473 sibships were detected.

\section{THE MIXED MODEL}

The mixed model (Morton and MacLean, 1974) postulates an underlying scale of liability, to which a major locus, a polygenic component, and environment contribute independently. A quantitative trait linearly related to liability may be measured, but whenever only information on affection status is available, as in the present study, mean and variance of liability are arbitrary and taken equal to zero and one, respectively; affection then is defined by a threshold on the liability scale, and the threshold is determined by incidence of affection, assumed to be known from other evidence. The major locus is assumed biallelic, producing three genotypes. The distance between the homozygous means on the liability scale is called displacement, $t$. The relative position of the heterozygous class is called the degree of dominance, $d$. By convention, if the heterozygote mean is near the lower homozygote the locus is called recessive, if near the higher one it is called dominant, and if in the middle, it is called additive. The relative sizes of the genotype classes are determined by the gene frequency, $q$, as panmixia is assumed. The polygenic and environmental factors are assumed independent and normally distributed. The proportions of the total variance $V$ resulting from the polygenic and the environmental contributions are denoted $H$ and $E$, respectively. The environmental contribution may be further partitioned into two components, environment common to sibs and random residual, accounting for proportions of the total variance $B$ and $R$, respectively. Variation in incidence with sex (if not accountable to a sex-linked major locus), or with birth order, can be treated by a shift of the liability scale, as suggested by Carter (1961).

SEGREGATION ANALYSIS UNDER THE MIXED MODEL

Population incidences are an input in segregation analysis; the same incidences are reported for Lon- 
don and Birmingham (McKeown et al., 1951a, Carter and Evans, 1969). In this latter reference, incidences have been estimated according to sex and birth order, and these estimates have been used for the present analysis (McKeown et al., 1951a, Table VIII). As birth order was not recorded for an important part of the data, incidences for various family sizes have been computed for each sex; overall incidence is 0.00478 in males, 0.00118 in females. Sex and birth order effects are taken into account in this analysis by shifting the liability scale, so that various thresholds are computed to fit the given incidences.

Since the sampling procedures of the several studies were quite different, the ascertainment probability was established separately for each subsample by analysis of distribution of probands among affected in all sibships, using the computer programme SEGRAN (Morton, 1969). The result

Table 1 Summary of input data

\begin{tabular}{lccc}
\hline Probability of ascertainment & \multicolumn{3}{l}{$\begin{array}{l}\text { Number of sibships for each } \\
\text { mating type }\end{array}$} \\
\cline { 2 - 4 } & $N{ }^{*} \times N \bigcirc$ & $A \sigma^{*} \times N ᄋ$ & $N{ }^{*} \times A+$ \\
\hline$\pi=1$ & 0 & $330(3)$ & $106(3)$ \\
$\pi=0.22$ & $963(3)$ & $3(3)$ & $1(3)$ \\
$\pi=0.36$ & $212(1)$ & 0 & 0 \\
$\pi=0.79$ & $473(2)$ & 0 & 0 \\
\hline
\end{tabular}

Incidence of pyloric stenosis according to sex and birth order (2)

\begin{tabular}{llllll}
\hline Sex & Overall & \multicolumn{4}{l}{ Birth ranks } \\
\cline { 3 - 6 } & & 1 & 2 & 3 & $4+$ \\
\hline Male & 0.00478 & 0.0064 & 0.0046 & 0.0041 & 0.0020 \\
Female & 0.00118 & 0.0019 & 0.0008 & 0.0007 & 0.0007 \\
\hline
\end{tabular}

Sources: (1) Cockayne and Penrose (1943); (2) McKeown et al. (1951a, b); (3) Carter and Evans (1969).

clearly indicated heterogeneity $\left(\chi_{2}^{2}=16 \cdot 3\right)$; therefore, separate maximum likelihood estimates were used in the segregation analysis, each for its respective subsample, as presented in Table 1.

The likelihood surfaces were also investigated separately because of the possibility of heterogeneity among the samples from different investi- gators. However, there proved to be striking simi- $\stackrel{\unrhd}{\circ}$ larity in likelihood among all three samples, and therefore likelihood evidence was pooled throughout the segregation analysis.

Preliminary investigation of the likelihood surface for various values of the parameters of the modelo pointed to negligible environment common to sibs. $\frac{\overline{0}}{\mathrm{C}}$ There was little information on dominance, and $\overline{\bar{n}}$ equivalent likelihoods, though for different values of $t$ and $q$, were obtained for values of the dominance parameter $d$ equal to $1,0.5$, and 0 , respectively. Hences in this analysis we have assumed no common environ- $\overrightarrow{0}$ ment to sibs $(B=0)$ and complete dominance $(d=1) . \overrightarrow{-}$

Three models of inheritance are considered: the $\bar{\omega}_{-}$ mixed model, the generalised single locus model, and 5 the multifactorial model. Those last two models cano be considered as special cases of the mixed model, $\vec{A}$ and, therefore, are alternative subhypotheses, con- $-\circ$ cerning the nullity of one or several parameters, that ${ }^{+}$ can be tested against the hypothesis that the mixedoo model holds.

Maximum likelihood estimation has been carriedout under the mixed model hypothesis and the two alternatives mentioned (Table 2), and likelihood ratio tests reveal that the mixed model fits the data 3 significantly better than the generalised single locus $\mathbb{D}$ model $\left(\chi_{1}^{2}=8.05\right)$. The multifactorial model yields alikelihood almost equal to that obtained under themixed model. This indicates that consideration ofa. major locus, in addition to polygenic and envire mental variation, is not necessary to account for thes observations and, by an argument of simplicity (e.g.o Ramsey, 1931), we shall retain the multifactoriala model as a description of the paitern of familial $\mathbb{Q}^{\mathbb{Q}}$ aggregation in pyloric stenosis.

The suggestion of a possible maternal effect led us $ঙ$ to question the homogeneity of estimates for different? mating types. No heterogeneity was found between mating types normal $\times$ normal and father affected $x$ mother normal $\left(\chi_{1}^{2}=0 \cdot 34\right)$, and they were subse quently pooled; however, as seen in Table 2 , there iso significant heterogeneity between those pooled matings and matings with father normal and motheró affected $\left(\chi_{1}^{2}=8 \cdot 46\right)$. Estimation restricted to matings with normal mothers leads again to adoption of theo multifactorial model to account for these data, and it

Table 2 Segregation analysis of pyloric stenosis

\begin{tabular}{|c|c|c|c|c|c|c|c|c|c|c|}
\hline & \multicolumn{4}{|l|}{ Mixed model } & \multicolumn{4}{|c|}{ Generalised single locus model } & \multicolumn{2}{|c|}{ Multifactorial model } \\
\hline & $D T \pm T_{\mathrm{T}}$ & $\boldsymbol{Q} \pm \sigma_{\boldsymbol{Q}}$ & $H \pm \sigma_{H}$ & $-2 \ln L+C$ & $D$ & $\boldsymbol{T} \pm \boldsymbol{\sigma}_{\boldsymbol{T}}$ & $\boldsymbol{Q} \pm \sigma_{\boldsymbol{Q}}$ & $-2 \ln L+C$ & $\boldsymbol{H} \pm \sigma_{\boldsymbol{H}}$ & $-2 \ln L+C$ \\
\hline $\begin{array}{l}\text { All matings } \\
N \sigma^{*} \times N \uparrow \text { or } A \sigma^{*} \times N \uparrow \\
N \delta^{*} \times A \%\end{array}$ & $\begin{array}{ll}1.0 & 1 \cdot 7 \pm 1.6 \\
1.0 & 1 \cdot 7 \\
1.0 & 2 \cdot 4\end{array}$ & $\begin{array}{l}0.001 \pm 0.007 \\
0.000 \pm 0.001 \\
0.001 \pm 0.003\end{array}$ & $\begin{array}{l}0.76 \pm 0.26 \\
0.79 \pm 0.12 \\
0.99 \pm 0.33\end{array}$ & $\begin{array}{r}-105 \cdot 21 \\
-262 \cdot 71 \\
148 \cdot 81\end{array}$ & $\begin{array}{l}1.0 \\
1.0 \\
1.0\end{array}$ & $\begin{array}{l}2 \cdot 2 \pm 0 \cdot 1 \\
2 \cdot 0 \pm 0 \cdot 1 \\
2 \cdot 8 \pm 0 \cdot 3\end{array}$ & $\begin{array}{l}0.002 \pm 0.001 \\
0.004 \pm 0.002 \\
0.001 \pm 0.001\end{array}$ & $\begin{array}{r}-97 \cdot 16 \\
-257 \cdot 66 \\
150 \cdot 62\end{array}$ & $\begin{array}{l}0.84 \pm 0.04 \\
0.79 \pm 0.04 \\
1.0 \pm 0.07\end{array}$ & $\begin{array}{r}-104 \cdot 84 \\
-262 \cdot 71 \\
149 \cdot 41\end{array}$ \\
\hline
\end{tabular}

Tests of hypotheses: $H=0$, over all matings: $\chi_{1}^{2}=8 \cdot 05$. Homogeneity of mating types father normal $\times$ mother normal and father affected $\times$ mother ${ }^{2}$ normal $: \chi_{1}^{2}=0 \cdot 34$. Homogeneity of mating types with mother normal and with mother affected: $\chi_{1}^{2}=8 \cdot 46$. 
was verified that simultaneous estimation of $B$, proportion of the variance caused by environment common to sibs, does not significantly improve the like$\operatorname{lihood}\left(\chi_{1}^{2}=2 \cdot 43\right)$.

Segregation analysis restricted to mating types with affected mothers yields the extreme estimate $H=1$ (Table 2) for the parameter of the multifactorial model, perhaps as a consequence of a maternal effect. Since this is a constrained estimate, values of $H$ above unity being inadmissible, it is unsuitable for prediction of risk. Rather than using such a boundary solution, we propose to account for a maternal effect as follows: we assume that the estimate $H=0.79$ holds, but that children of affected mothers have a higher mean liability than others, and the maternal effect revealed in the offspring of affected mothers is accounted for, if not explained, by a shift of the liability scale in children of those mothers. This shift is determined by finding that multiplier of the incidences among children which maximises the likelihood for these matings, subject to the condition $H=0.79$. The estimate of this multiplicative factor is 2.9 , which is appropriate whether the maternal effect is the result of stress, gastrin secretion, or other physiological mechanism, or merely the result of more liberal diagnosis of pyloric stenosis in the children of affected women.

\section{Recurrence risks for pyloric stenosis}

Recurrence risks for pyloric stenosis can be computed for various family histories with the programme RISK (see Appendix), given the present estimate of the parameter of the multifactorial model, $H=0.79$, and incidences according to sex and birth order already mentioned. In the present situation a tabulation of risk figures for various family histories is impracticable, and recurrence risks are best computed for every specific family with the help of a computer programme. The following examples are given merely as an illustration of how risks vary with family history. In each case, risk is given for an individual of unknown sex, together with, in parentheses, risk for a male and a female, respectively.

For parents of unknown rank, risk for a first born affected is $0.003(0.005,0.001)$ for normal parents, $0.051(0.073,0.029)$ if the father was affected, and $0.144(0.199,0.090)$ if the mother was affected. After a first born male affected, the risks are $0.028(0.045$, $0.011), 0.098(0.141,0.045)$, and $0.183(0.271,0.096)$ for these three respective mating types; after a firstborn female affected, they are $0.037(0.060,0.015)$, $0 \cdot 111(0 \cdot 167,0.056)$ and $0.213(0.310,0 \cdot 116)$, respectively. After both first born and second born affected, the risk for a third born varies from $0.070(0 \cdot 109$, $0.031)$ to $0.306(0.428,0.185)$ if the children were males with normal parents and females with mother affected, respectively. The range of the possible values of the risk is still larger if one considers situations where affected occupy various birth ranks. As expected (e.g. Smith, 1971), normal children contribute per se little information (the risk for a fifth

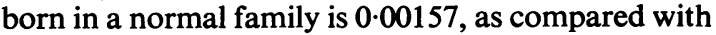
an a priori risk of 0.002 ), but for normal parents with one affected male among five children with alternating sex, risk for a sixth born is $0.013(0.019,0.008)$ or $0.016(0.024,0.010)$ depending on whether the affected was first born or fifth born. Both risks are lower than for a second born after a first born male affected with normal parents, $0.028(0.045,0.011)$, and this is mostly a consequence of the birth order effect. Moreover, if one were to ask, among families with normal parents, what is the probability that the first born were affected, given that a younger brother is affected, one would find a probability of 0.044 $(0.064,0.024)$ if this younger brother were second born, but $0.053(0.093,0.038)$ if he were fourth born, other sibs being of unknown status. This illustrates that empirical risks based on pairs of relatives are

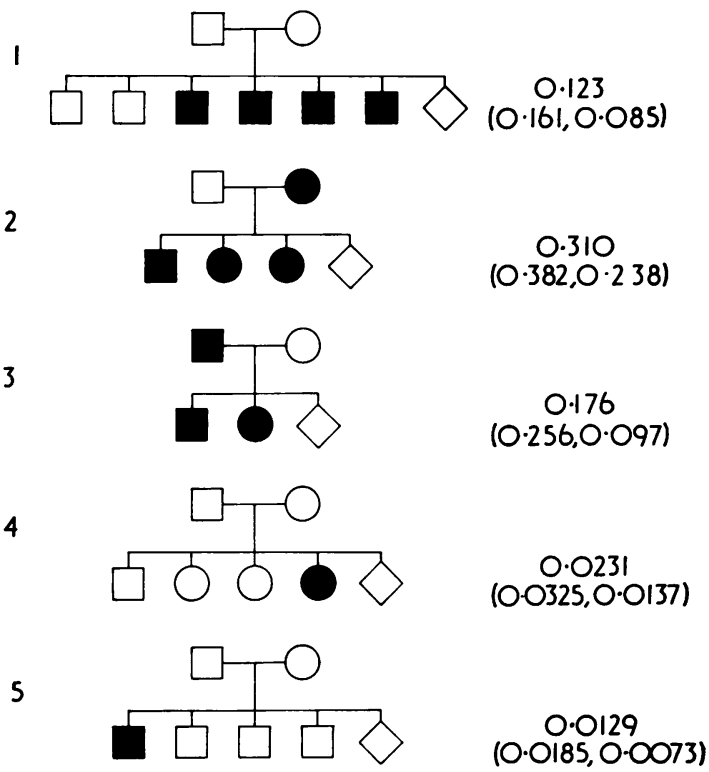

Fig. Specific recurrence risks for certain families. $\square$, $\circ$ normal; $\square, \bullet:$ affected; $\diamond:$ individual at risk. For each family, recurrence risk is given for an individual of unknown sex, together with the risks when sex is male or female respectively. Sources-1: Gailey (1948), as reported in Cameron (1955); 2: Carter and Evans (1969), family No. 352; 3: Carter and Evans (1969), family No. 152; 4: McKeown et al. (1951b), family No. 72; 5: McKeown et al. (1951b), family No. 333. 
inappropriate in the presence of a monotonic birth order effect, and specific risks should be computed for particular family histories.

A few examples concerning some families reported in the literature are given in the Figure.

\section{Discussion}

Segregation analysis of nuclear families yields more support for the multifactorial model than other alternatives as a description of patterns of familial aggregation in pyloric stenosis, and reveals heterogeneity among mating types in agreement with a suggested maternal effect (Carter, 1972; Dodge, 1972a, 1974; Kidd and Spence, 1976).

However complex the mixed model, it may be argued that it is still too simplistic, particularly in two respects: non-additive polygenic variation is neglected, and no allowance is made for non-genetic parent-offspring correlation. A first consequence is that the degree of genetic determination cannot be estimated; but, as pointed out by Falconer (1965), the heritability, expressing the extent to which the phenotypes exhibited by parents are transmitted to their offspring, is more relevant in a genetic counselling context. A second consequence is that an estimate of heritability might be inflated by nongenetic sources of parent-offspring correlations to an unknown extent.

Dominance deviation can simulate an effect of environment common to sibs (MacLean et al., 1975), but as simultaneous estimation of $H$ and $B$ does not improve significantly the likelihood, both effects may be reasonably considered negligible here.

At least two sources of variation could simulate a maternal effect. Since incidence of affection is lower among females than among males, affected mothers would be of the more extreme genotype more often than males under the mixed model; however, we have seen that these data do not support the hypothesis of a major gene with or without polygenic variation. More thorough detection of affection may have occurred in the follow-up of the offspring of affected parents, but this is not supported by the test of homogeneity between normal $\times$ normal matings and father affected $\times$ mother normal matings. Because the sample with an affected mother is small and the maternal effect is not understood, prediction of risks in such families may be less reliable than in the great majority of families with a normal mother.

Our conclusion that the multifactorial model best accounts for the observed familial aggregation of pyloric stenosis is in contradiction with the result presented in a recent paper in this journal (Kidd and Spence, 1976), where the multifactorial model was rejected in favour of the generalised single locus model. However, the analysis of these authors involved a stringent reduction of familial data to pairs of relatives over different mating types and various ascertainments, hence accordingly losing information and increasing noise.

More information could theoretically be used by the method of pedigree analysis (Elston and Stewart, 1971), though in practice ascertainment problems and heterogeneity of diagnosis may counterbalance the increased amount of information expected under this approach.

Consideration of twin data has often been advocated as an aid in the determination of the mode of inheritance of familial diseases. However, three important difficulties occur in connection with twin data. Because of the rarity of twinning, collection of a large series of homogeneous and reliable data may be extremely difficult; determination of zygosity is liable to misclassification; environmental effects in utero are likely to be different between monozygotic and dizygotic twins, and possibly also between monoand dichorionic twins. A study by Metrakos (1953) is instructive in this context: of 132 twin pairs in the literature, only 47 satisfy strict criteria of diagnosis of affection as well as zygosity. Of these, 12/18 (66\%) were monzygotic concordant twins, interestingly similar to the proportion of monochorionic twins among monozygotic twins (Bulmer, 1970), and 1/28 $(3.5 \%)$ were dizygotic concordant twins. At most this suggests that risk among dizygotic twins does nō significantly differ from that of sibs, and uterine effects would be required to explain the observed concordance among monozygotic twins, as a concordance rate around $25 \%$ would be expected when the present estimates of incidence and heritability are used.

There is much to be learned about the aetiology of pyloric stenosis, especially the rare cases in monozygotic twins or from affected mothers, but for the great majority of cases present evidence is adequate to provide reliable and specific recurrence risks.

\section{Appendix}

CALCULATIONS OF RECURRENCE RISKS UNDER THE MIXED MODEL

Recurrence risks are calculated from specific information together with estimates of the parameters under that model which best fits data on familial distribution. The basic risk calculation is the probability that an unknown child be affected given all the phenotype information of the family:

$$
P\left(a f \mid \phi_{k}, \phi_{p}\right)
$$

where af signifies affection, $\phi_{k}$ is the phenotype of all known children, and $\phi_{p}$ is the phenotype of known 
parents. The likelihood calculated is the probability of the phenotypes of the children given the phenotypes of the parents:

$$
P\left(\phi_{k} \mid \phi_{p}\right)=\int_{v} \sum_{i, j} P\left(\phi_{k} \mid i, j, v\right) P\left(i, j, v \mid \phi_{p}\right) .
$$

In the first term of this conditional expression, each child is statistically independent of the phenotypes of his sibs and his parents. His probability depends only upon the genetic parameters: $i, j$ for the parental genotypes at the major locus and $v$ for the polygenic contribution. The segregation analysis programme NUCLEAR (MacLean et al., 1975) has been extended in the programme SHIFT (MacLean, unpublished) to permit a discontinuous liability indicator, here defined by sex and birth order, which displaces mean liability to conform to specific incidence.

The same important conditional independence holds for the unknown child at risk. This fact makes the risk calculation a very simple extension of the likelihood calculation. We have:

$$
P\left(a f \mid \phi_{k}, \phi_{p}\right)=\int_{v} \sum_{i, j} P(a f \mid i, j, v) P\left(i, j, v \mid \phi_{k}, \phi_{p}\right)
$$

and further

$$
P\left(i, j, v \mid \phi_{k}, \phi_{p}\right)=P\left(\phi_{k}, i, j, v \mid \phi_{p}\right) / P\left(\phi_{k} \mid \phi_{p}\right)
$$

where

$$
P\left(\phi_{k}, i, j, v \mid \phi_{p}\right)=P\left(\phi_{k} \mid i, j, v\right) P\left(i, j, v \mid \phi_{p}\right) .
$$

Explicit expressions of these probabilities in terms of the parameters of the mixed model can be found in Morton and MacLean (1974).

The computer programme RISK (MacLean, unpublished) has been written to perform these computations. Other outputs of this programme are the conditional probability for each genotype $\boldsymbol{P}\left(g_{i} \mid\right.$ Family $)$, likelihood of family, standard deviation of risk, and tolerances of risk. These later outputs summarise the variability of the risk predicted over families of same composition, and their usefulness in counselling situations is uncertain. The $x \%$ tolerance of a family is the probability that a family has a risk greater than $x \%$, by analogy with the common statistical concept, and is, therefore, a monotonically decreasing function of risk over the range $[0,1]$. Tolerance is given by

$$
T=\int_{v} \sum_{i, j \in A} P\left(\phi_{k} \mid \phi_{p}, i, j, v\right) d v
$$

where $A$ is the subspace of all points $i, j$ for major locus and $v$ for polygenic variables for which family risk exceeds the tolerance limit.

\section{References}

Beckman, L. (1969). Blood groups and serum lipids and phosphatases. Lancet, $2,799$.

Bruckner, W. L., Snow, H. D., and Fonkalsrud, E. W. (1970).
Gastrin secretion in the canine fetus following maternal stimulation: experimental studies on placental transfer of insulin, histamine and gastrin. Surgery, 67, 360-363.

Bulmer, M. G. (1970). The Biology of Twinning in Man. Clarendon Press, Oxford.

Cameron, A. L. (1955). Familial occurrence of congenital hypertrophic pyloric stenosis. Archives of Surgery, 70, 877894.

Carter, C. O. (1961). The inheritance of congenital pyloric stenosis. British Medical Bulletin. 17, 251-254.

Carter, C. O. (1972). Genetics of infantile pyloric stenosis. Birth Defects: Original Article Series, Vol. VIII, No. 2, 12-14.

Carter, C. O. (1976). Genetics of common single malformations. British Medical Bulletin, 32, 21-26.

Carter, C. O., and Evans, K. A. (1969). Inheritance of congenital pyloric stenosis. Journal of Medical Genetics, 6, 233254.

Carter, C. O., and Powell, B. W. (1954). Two-generation pyloric stenosis. Lancet, 1, 746.

Cockayne, E. A., and Penrose, L. S. (1943). The genetics of congenital pyloric stenosis. Ohio Journal of Science, 43, 1-16.

Craig, W. S. (1955). Palpable contractile pyloric tumors in the newly born. Archives of Disease in Childhood, 30, 484-492.

Dodge, J. A. (1970). Production of duodenal ulcers and hypertrophic pyloric stenosis by administration of pentagastrin to pregnant and newborn dogs. Nature (London), 225, 284-285.

Dodge, J. A. (1972a). Psychosomatic aspects of infantile pyloric stenosis. Journal of Psychosomatic Research, 16, 1-5.

Dodge, J. A. (1972b). Infantile pyloric stenosis: a multifactorial condition. Birth Defects: Original Article Series, Vol. VIII, No. 2, 15-21.

Dodge, J. A. (1974). Maternal factor in infantile hypertrophic pyloric stenosis. Archives of Disease in Childhood, 49, 825.

Dodge, J. A. (1975). Infantile hypertrophic pyloric stenosis in Belfast, 1957-1969. Archices of Disease in Childhood, 50, 171-178.

Elston, R. C., and Stewart, J. (1971). A general model for the genetic analyses of pedigree data. Human Heredity, 21, 523-542.

Falconer, D. S. (1965). The inheritance of liability to certain diseases, estimated from the incidence among relatives. Annals of Human Genetics, 29, 51-76.

Gerrard, J. W., Waterhouse, A. H., and Maurice, D. G. (1955). Infantile pyloric stenosis. Archives of Disease in Childhood, 30, 493 .

Heinsisch, H. M. (1967). Die sog pylorus hypertrophie in tierversuch. Klinische Wochenschrift, 45, 1251-1252.

Heuch, I., and Li, F. H. F. (1972). PEDIG-A computer program for calculation of genotype probabilities using phenotype information. Clinical Genetics, 3, 501-504.

Holt, E. (1917). Hypertrophic stenosis in infants. Journal of the American Medical Association, 68, 1517-1524.

Karim, A. A., Morrison, J. E., and Parks, T. G. (1974). The role of pentagastrin in the production of canine hypertrophic pyloric stenosis and pyloroduodenal ulceration. British Journal of Surgery, 61, 327.

Kidd, K. K., and Spence, M. A. (1976). Genetic analyses of pyloric stenosis suggesting a specific maternal effect. Journal of Medical Genetics, 13, 290-294.

Leck, I. (1976). Descriptive epidemiology of common malformations (excluding central nervous system defects). British Medical Bulletin, 32, 45-52.

McKeown, T., MacMahon, B., and Record, R. G. (1951a). The incidence of congenital pyloric stenosis related to birth rank and maternal age. Annals of Human Genetics, 16, 249259. 
McKeown, T., MacMahon, B., and Record, R. G. (1951b). The familial incidence of congenital pyloric stenosis. Annals of Human Genetics, 16, 260-281.

MacLean, C. J., Morton, N. E., and Lew, R. (1975). Analysis of family resemblance. IV. Operational characteristics of segregation analysis. American Journal of Human Genetics, 27,365-384.

Metrakos, J. D. (1953). Congenital hypertrophic pyloric stenosis in twins. Archives of Disease in Childhood, 28, 351358.

Morris, N. (1968). Attitude survey in pregnancy. Journal of Psychosomatic Research, 12, 83-84.

Morton, N. E. (1969). Segregation analysis. In Computer Applications in Genetics, pp. 129-139. Ed. by N. E. Morton. University of Hawaii Press, Honolulu.

Morton, N. E., and MacLean, C. J. (1974). Analysis of family resemblance. III. Complex segregation of quantitative traits. American Journal of Human Genetics, 26, 489-503.

Murphy, E. A., and Mutalik, G. S. (1969). The application of Bayesian methods in genetic counselling. Human Heredity, 19, 126-151.
Ramsey, F. P. (1931). The Foundations of Mathematics and Other Logical Essays. Kegan Paul, London; Harcourt Brace, New York.

Shim, W. K. T., Campbell, A., and Wright, S. W. (1970). 276 cases of pyloric stenosis in Hawaii: II. Racial aspects. Hawaii Medical Journal, 29, 292-295.

Smith, C. (1971). Recurrence risks in multifactorial inheritance. American Journal of Human Genetics, 23, 578-588.

Thomson, W. A., and Gaisford, W. F. (1935). Congenital pyloric stenosis. British Medical Journal, 2, 1037-1041.

Wallgren, A. (1960). Is the rate of hypertrophic stenosis $\mathbb{Q}$ declining? Acta Paediatrica, 49, 530-535.

Requests for reprints to Dr J. M. Lalouel, Population Genetics Laboratory, University of Hawaii at Manoa, 1980 East-West Road, Honolulu, Hawaiiọ 96822. 